\title{
Engaging the terminal: highlighting routes for promoting non-covalent interactions with uranyl oxo atoms
}

\author{
Korey P. Carter, Mark Kalaj, and Christopher L. Cahill* \\ Department of Chemistry, The George Washington University, Washington, DC, \\ 20052, USA \\ koreypcarter@gwmail.gwu.edu
}

Harnessing the nominally terminal oxo atoms of the linear uranyl $\left(\mathrm{UO}_{2}{ }^{2+}\right)$ cation represents both a significant challenge and opportunity within the field of f-element hybrid materials. We have developed multiple approaches for promoting oxo atom participation in halogen-oxo and cation-cation interactions via synthetic strategies based on the judicious selection of halogen atoms and selected transition metal (TM) cations. These synthesis efforts have yielded a diverse suite of hybrid materials including uranyl molecular complexes, coordination polymers, and heterometallic hybrid materials, which have all been characterized via single crystal X-ray diffraction, Raman, Infrared (IR), and luminescence spectroscopy. Raman and IR spectroscopy results are used to generate stretching force and interaction force constants, which indicate that both halogen-oxo and cation-cation interactions weaken the $\mathrm{U}=\mathrm{O}$ bond. Presented will be an overview of the routes that yield oxo participation in non-covalent interactions along with the relevant spectroscopy data that highlight our ongoing efforts to enhance structure-property delineations in this area at the frontier of uranyl crystal chemistry. 